\title{
Relatively dense sets, corrected uniformly almost periodic functions on time scales, and generalizations
}

\author{
Chao Wang ${ }^{1 *}$ (1) and Ravi P Agarwal ${ }^{2,3}$
}

\section{"Correspondence:}

chaowang@ynu.edu.cn

'Department of Mathematics,

Yunnan University, Kunming,

Yunnan 650091, People's Republic

of China

Full list of author information is

available at the end of the article

\begin{abstract}
In this paper, we use the analysis of relatively dense sets to point out some deficiencies and inaccuracies in the definition of uniformly almost periodic functions which has been proposed in recent works, and we correct it. Some new generalizations of invariance under translation time scales and almost periodic functions are established. Our study will ensure that now we can study almost periodic problems precisely on time scales.
\end{abstract}

MSC: $26 \mathrm{E} 70 ; 43 \mathrm{~A} 60$

Keywords: time scales; relatively dense set; uniformly almost periodic functions

\section{Problems and corrections}

Nowadays, the study of dynamic equations on time scales is a leading topic of research in different directions including directions for boundary value problems, periodic and almost periodic problems (see [1-7]), etc. As one of the most important research directions, the occurrence of almost periodic phenomenon is very common in nature. Thus the existence of almost periodic solutions for a wide variety of dynamical systems has been studied extensively. In 2011, the authors of [8,9] extended this concept to uniformly almost periodic functions on time scales. This concept has been extensively used to study almost periodic solutions of functional dynamic equations, neural networks, biological dynamic models, and so on. However, the basic concept of uniformly almost periodic functions on time scales introduced in $[8,9]$ is not accurate. Unfortunately, in several recent works [10-18] this false concept has been cited and applied.

The main purpose of this paper is to point out the deficiencies and inaccuracies in the works of $[8,9]$, and then to give a correct definition of uniformly almost periodic functions. We begin with the following basic definitions.

Definition 1.1 $([8,19])$ A time scale $\mathbb{T}$ is called invariant under a translation time scale if

$$
\Pi:=\{\tau \in \mathbb{R}: t \pm \tau \in \mathbb{T}, \forall t \in \mathbb{T}\} \neq\{0\}
$$

Definition 1.2 The set $\Pi$ in Definition 1.1 is called an invariant translation set for $\mathbb{T}$.

(0) 2015 Wang and Agarwal. This article is distributed under the terms of the Creative Commons Attribution 4.0 International License (http://creativecommons.org/licenses/by/4.0/), which permits unrestricted use, distribution, and reproduction in any medium, provided you give appropriate credit to the original author(s) and the source, provide a link to the Creative Commons license, and indicate if changes were made. 
Remark 1.1 It follows from Definition 1.1 that $\Pi$ is also a closed subset of $\mathbb{R}$, so $\Pi$ is a time scale.

Definition 1.3 (Problem 1, Definition 3.7 in [8]) Let $\mathbb{T}$ be an invariant under a translation time scale. A function $f \in C\left(\mathbb{T} \times D, \mathbb{E}^{n}\right)$ is called an almost periodic function in $t \in \mathbb{T}$ uniformly for $x \in D$ if the $\varepsilon$-translation set of $f$,

$$
E\{\varepsilon, f, S\}=\{\tau \in \Pi:|f(t+\tau, x)-f(t, x)|<\varepsilon, \text { for all }(t, x) \in \mathbb{T} \times S\}
$$

is a relatively dense set in $\mathbb{T}$ for all $\varepsilon>0$ and for each compact subset $S$ of $D$, that is, for any given $\varepsilon>0$ and each compact subset $S$ of $D$, there exists a constant $l(\varepsilon, S)>0$ such that each interval of length $l(\varepsilon, S)$ contains a $\tau(\varepsilon, S) \in E\{\varepsilon, f, S\}$ such that

$$
|f(t+\tau, x)-f(t, x)|<\varepsilon, \quad \text { for all }(t, x) \in \mathbb{T} \times S .
$$

Here, $\tau$ is called the $\varepsilon$-translation number of $f$ and $l(\varepsilon, S)$ is called the inclusion length of $E\{\varepsilon, f, S\}$.

Note that the above definition requires $E\{\varepsilon, f, S\}$ to be a relatively dense set in $\mathbb{T}$, which makes Definition 1.3 inaccurate and false. To justify our claim, we will give a detailed explanation and provide a counter-example. For this, we recall the concept of a relatively dense set.

Definition 1.4 (Fink (1974) from [20]) Let $A \subset B \subset \mathbb{R}$, we say that $A$ is relatively dense in $B$ if there exists a positive number $l$ such that for all $a \in B$ we have $[a, a+l]_{B} \cap A \neq \emptyset$, where $[a, a+l]_{B}=[a, a+l] \cap B$, and $l$ is called the inclusion length.

Let $\mathbb{T}=B$ in Definition 1.4, then the concept of relative density in $\mathbb{T}$ from Definition 1.4 can be stated as follows.

Definition 1.5 (Problem 2) Let $A \subset \mathbb{T}$, we say that $A$ is relatively dense in $\mathbb{T}$ if there exists a positive real number $l$ such that for all $a \in \mathbb{T}$ we have $[a, a+l]_{\mathbb{T}} \cap A \neq \emptyset$, here $l$ is called the inclusion length.

Definition 1.5 is directly used to introduce Definition 1.3. However, this definition is not accurate. In fact, to some extent, false. To explain this, we claim that the closed interval $[a, b]_{\mathbb{T}}$ must have $a, b \in \mathbb{T}$. Recall that the definition of a Cauchy integral on time scales (see Definition 1.71 in [21]) is completely based on this fact.

Definition 1.6 (Bohner (2001) from [21]) Let $F$ be a pre-antiderivative of $f$. Then the Cauchy integral on $\mathbb{T}$ is defined by

$$
\int_{a}^{b} f(t) \Delta t=F(a)-F(b), \quad \text { for all } a, b \in \mathbb{T} .
$$

Needless to say, this basic definition plays a very important role in the calculus on time scales. If this definition is violated, then the whole theory on time scales will collapse. 
However, there exist many integrals in [8] such as the following:

$$
\int_{t}^{t+l} f(s) \Delta s, \quad \text { where } l \in \mathbb{R} \text { is an inclusion length, }
$$

but $t \in \mathbb{T}$ cannot guarantee that $t+l \in \mathbb{T}$, where $l \in \mathbb{R}$ is an inclusion length in Definition 1.5 .

We also note that, in Definition 1.3, it is required that $E\{\varepsilon, f, S\} \subset \Pi$ is relatively dense in $\mathbb{T}$, which means that the set $A=E\{\varepsilon, f, S\}$ in Definition 1.5 is relatively dense. But then in Definition 1.5, we need $[a, a+l]_{\mathbb{T}} \cap E\{\varepsilon, f, S\} \neq \emptyset$, which implies that $[a, a+l]_{\mathbb{T}} \cap \Pi \neq \emptyset$, and hence $\mathbb{T} \cap \Pi \neq \emptyset$. However, this condition is too restrictive. In fact, the following counterexample shows that $\mathbb{T} \cap \Pi=\emptyset$.

Example 1.2 Consider the following time scale where $a, b>0$ :

$$
\mathbb{P}_{a, b}=\bigcup_{k=-\infty}^{\infty}[(2 k+1)(a+b),(2 k+1)(a+b)+a] .
$$

Clearly,

$$
\sigma(t)= \begin{cases}t, & \text { if } t \in \bigcup_{k=-\infty}^{\infty}[(2 k+1)(a+b),(2 k+1)(a+b)+a), \\ t+a+2 b, & \text { if } t \in \bigcup_{k=-\infty}^{\infty}\{(2 k+1)(a+b)+a\}\end{cases}
$$

and

$$
\mu(t)= \begin{cases}0, & \text { if } t \in \bigcup_{k=-\infty}^{\infty}[(2 k+1)(a+b),(2 k+1)(a+b)+a), \\ a+2 b, & \text { if } t \in \bigcup_{k=-\infty}^{\infty}\{(2 k+1)(a+b)+a\} .\end{cases}
$$

Note that here we can consider $\Pi=\{2 n(a+b), n \in \mathbb{Z}\}$. But then

$$
2 n a+(2 n-1) b<2 n(a+b)<(2 n+1)(a+b), \quad \text { where } a, b>0, n \in \mathbb{Z},
$$

which implies that $2 n(a+b) \notin \mathbb{T}$ for all $n \in \mathbb{Z}$, that is, $\mathbb{T} \cap \Pi=\emptyset$.

Remark 1.3 In Example 1.2, let $a=b=1$, to obtain the time scale

$$
\mathbb{P}_{1,1}=\bigcup_{k=-\infty}^{\infty}[4 k+2,4 k+3]
$$

Clearly $4 n \notin \mathbb{P}_{1,1}$ for $n \in \mathbb{Z}$. Since $\Pi=\{4 n, n \in \mathbb{Z}\}$, we have $\mathbb{T} \cap \Pi=\emptyset$.

Remark 1.4 From Example 1.2 and Remark 1.3, it follows that the invariant translation set $\Pi$ for $\mathbb{T}$ may be separated from $\mathbb{T}$, i.e., $\mathbb{T} \cap \Pi=\emptyset$. Furthermore, the time scale $\mathbb{T}$ from Example 1.2 is periodic, which means that Definition 1.3 does not include the situation for periodic time scales like Example 1.2. Hence, to some extent, Definition 1.3 is false.

From Example 1.2 and Remark 1.3, it is clear that it is too particular if we require that $E\{\varepsilon, f, S\}$ is relatively dense in $\mathbb{T}$ in Definition 1.3 because it implies that $\mathbb{T} \cap \Pi \neq \emptyset$. Through 
our investigations, we find that the relatively dense set should not be for $\mathbb{T}$ rather it should be for the set $\Pi$ defined in Definition 1.1.

Definition 1.7 (Correction of Definition 1.5) Let $\Pi$ be as in Definition 1.1 for an invariant under a translation time scale. Let $A \subset \Pi$, we say that $A$ is relatively dense in $\Pi$ if there exists a positive number $l \in \Pi$ such that for all $a \in \Pi$ we have $[a, a+l]_{\Pi} \cap A \neq \emptyset$, here $l$ is called the inclusion length.

Remark 1.5 It is clear that if $\tau_{1}, \tau_{2} \in \Pi$, then $\tau_{1} \pm \tau_{2} \in \Pi$. From this, we find that $a+l \in \Pi$ for any $a, l \in \Pi$. With this change all the deficiencies in the literature can be corrected (it suffices to note that for any $t \in \mathbb{T}, l \in \Pi$, we have $t+l \in \mathbb{T}$ ). Thus the integral

$$
\int_{t}^{t+l} f(s) \Delta s, \quad \text { where } t \in \mathbb{T}, l \in \Pi
$$

is now well defined for all $t \in \mathbb{T}$. This means that the inclusion length $l$ should be taken from the set $\Pi$.

Using Definition 1.7 and Remark 1.5, we can introduce a precise definition of uniformly almost periodic functions on time scales as follows.

Definition 1.8 (Correction of Definition 1.3) Let $\mathbb{T}$ be an invariant under a translation time scale. A function $f \in C\left(\mathbb{T} \times D, \mathbb{E}^{n}\right)$ is called an almost periodic function in $t \in \mathbb{T}$ uniformly for $x \in D$ if the $\varepsilon$-translation set of $f$,

$$
E\{\varepsilon, f, S\}=\{\tau \in \Pi:|f(t+\tau, x)-f(t, x)|<\varepsilon, \text { for all }(t, x) \in \mathbb{T} \times S\}
$$

is a relatively dense set in $\Pi$ for all $\varepsilon>0$ and for each compact subset $S$ of $D$, that is, for any given $\varepsilon>0$ and each compact subset $S$ of $D$, there exists a constant $l(\varepsilon, S)>0$ such that each interval of length $l(\varepsilon, S)$ contains a $\tau(\varepsilon, S) \in E\{\varepsilon, f, S\}$ such that

$$
|f(t+\tau, x)-f(t, x)|<\varepsilon, \quad \text { for all }(t, x) \in \mathbb{T} \times S .
$$

Here, $\tau$ is called the $\varepsilon$-translation number of $f$ and $l(\varepsilon, S)$ is called the inclusion length of $E\{\varepsilon, f, S\}$.

\section{Local-uniformly almost periodic functions}

In what follows we shall introduce a generalized concept of uniformly almost periodic functions on time scales, and we present some interesting results.

For convenience, we denote $\mathbb{T}^{\tau}=\{t+\tau: t \in \mathbb{T}\}$. If we choose a nonzero real number $\tau \in \Pi$, then it follows that $\mathbb{T}=\mathbb{T}^{\tau}$ if and only if $\mathbb{T}$ is invariant under translations, i.e., $\mathbb{T}$ coincides exactly with $\mathbb{T}^{\tau}$ if $\mathbb{T}$ is invariant under translations. Thus, Definition 1.1 has the following equivalent form.

Definition 2.1 A time scale $\mathbb{T}$ is called invariant under a translation time scale if

$$
\Pi:=\left\{\tau \in \mathbb{R}: \mathbb{T} \cap \mathbb{T}^{ \pm \tau}=\mathbb{T}\right\} \neq\{0\} .
$$


The following result guarantees that for any arbitrary time scale $\mathbb{T}$, there exists at least one invariant under a translation time scale $\mathbb{T}_{0 *} \subset \mathbb{T}$.

Theorem 2.1 Let $\tilde{\Pi}:=\left\{\tau \in \mathbb{R}: \mathbb{T} \cap \mathbb{T}^{\tau} \neq \emptyset\right\} \neq\{0\}$ and $\mathbb{T} \cap \mathbb{T}^{\tau}:=\mathbb{T}_{0}^{\tau}$, if $\mathbb{T}_{0 *}:=\bigcap_{\tau \in \tilde{\Pi}} \mathbb{T}_{0}^{\tau} \neq \emptyset$, then $\mathbb{T}_{0 *}$ is an invariant under a translation time scale.

Proof We construct the following family of sets:

$$
\mathscr{C}=\left\{\bigcap_{\tau \in A} \mathbb{T}_{0}^{\tau}: A \subset \tilde{\Pi}\right\} .
$$

Obviously, $\mathbb{T}_{0 *} \neq \emptyset$ implies that $\mathbb{T}_{0 *}$ is the minimal element in the family of sets $\mathscr{C}$. For contradiction, assume that there exists a $\tau_{0} \in \Pi$ such that $\mathbb{T}_{0 *} \cap \mathbb{T}_{0 *}^{\tau_{0}} \in \mathscr{C}$. We will have a contradiction if $\mathbb{T}_{0 *} \cap \mathbb{T}_{0 *}^{\tau_{0}} \subset \mathbb{T}_{0 *}$, because $\mathbb{T}_{0 *}$ is the minimal element in $\mathscr{C}$. But then $\mathbb{T}_{0 *} \cap \mathbb{T}_{0 *}^{\tau_{0}} \neq \emptyset$ implies that $\mathbb{T}_{0 *} \cap \mathbb{T}_{0 *}^{\tau_{0}}=\mathbb{T}_{0 *}$. Hence, $\mathbb{T}_{0 *}$ is an invariant under a translation time scale. This completes the proof.

Remark 2.2 According to Theorem 2.1, we know that $\mathbb{T}_{0 *}$ is a periodic time scale, i.e., there exists $\hat{\tau} \neq 0, \hat{\tau} \in \tilde{\Pi}$ such that $t+\hat{\tau} \in \mathbb{T}_{0 *} \subset \mathbb{T}$. We denote $\hat{\Pi}=\{n \hat{\tau}: n \in \mathbb{Z}\} \subset \tilde{\Pi}$ the invariant translation set for $\mathbb{T}_{0 *}$.

Now we state Zorn's lemma which will be needed to prove an interesting theorem.

Lemma 2.3 ([22], Zorn's Lemma) Suppose $(P, \preceq)$ is a partially ordered set. A subset $T$ is totally ordered iffor any $s, t$ in $T$ we have $s \preceq t$ or $t \preceq s$. Such a set $T$ has an upper bound $u$ in $P$ if $t \leq u$ for all $t$ in $T$. Suppose a non-empty partially ordered set $P$ has the property that every non-empty chain has an upper bound in $P$. Then the set $P$ contains at least one maximal element.

Theorem 2.4 Let $\mathbb{T}$ be an arbitrary time scale with $\sup \mathbb{T}=+\infty$, inf $\mathbb{T}=-\infty$. If $\mu: \mathbb{T} \rightarrow \mathbb{R}^{+}$ is bounded, then $\mathbb{T}$ contains at least one invariant under the translation unit.

Proof We denote the set

$$
\mathbb{I}=\overline{\left\{\mathbb{T} \cap \mathbb{T}^{\tau}: \tau \in[-\bar{\mu}, \bar{\mu}] \backslash\{0\}\right\}},
$$

where $\bar{A}$ denotes the closure of the set $A$ and $\bar{\mu}=\sup _{t \in \mathbb{T}} \mu(t)$. Clearly, I forms a semiordered set with respect to the inclusion relation, and $\mathbb{I}$ is closed. Denote $\mathbb{I}^{*}$ the any subset of $\mathbb{I}$ and is totally ordered. Hence, we can obtain two cases.

Case (1):

$$
\mathbb{I}^{*}=\left\{\left(\mathbb{T} \cap \mathbb{T}^{\tau_{n}}\right) \in \mathbb{I}: \mathbb{T}_{\tau_{n}} \supset \mathbb{T}_{\tau_{n+1}}, n \in \mathbb{N}\right\},
$$

then $\mathbb{T}_{\tau_{1}} \in \mathbb{I}^{*} \subset \mathbb{I}$ and $\mathbb{T}_{\tau_{1}}$ is an upper bound of $\mathbb{I}^{*}$ in $\mathbb{I}$.

Case (2):

$$
\mathbb{I}^{*}=\left\{\left(\mathbb{T} \cap \mathbb{T}^{\tau_{n}}\right) \in \mathbb{I}: \mathbb{T}_{\tau_{n}} \subset \mathbb{T}_{\tau_{n+1}}, n \in \mathbb{N}\right\},
$$


then $\lim _{n \rightarrow \infty}\left(\mathbb{T} \cap \mathbb{T}^{\tau_{n}}\right)=\bigcup_{n=1}^{\infty}\left(\mathbb{T} \cap \mathbb{T}^{\tau_{n}}\right)=\mathbb{T} \cap \mathbb{T}^{\tau_{\infty}} \subset \mathbb{T}$ is an upper bound of $\mathbb{I}^{*}$. Because $\mathbb{I}$ is closed, then $\mathbb{T} \cap \mathbb{T}^{\tau_{\infty}} \in \mathbb{I}$. According to Zorn's lemma (i.e., Lemma 2.3), there exists some $\tau_{0} \in[-\bar{\mu}, \bar{\mu}] \backslash\{0\}$ such that $\mathbb{T} \cap \mathbb{T}^{\tau_{0}}$ is the maximum element in $\mathbb{I}$. Note that since $\mu$ is bounded, $\mathbb{T} \cap \mathbb{T}^{\tau_{0}} \neq \emptyset$, and $\sup \left(\mathbb{T} \cap \mathbb{T}^{\tau_{0}}\right)=+\infty$, inf $\left(\mathbb{T} \cap \mathbb{T}^{\tau_{0}}\right)=-\infty$.

We can find a time scale $\mathbb{T}_{0}^{1}$ such that $\mathbb{T}_{0}^{1} \subset \mathbb{T}$ is the largest periodic sub-time scale in $\mathbb{T}$. For this, we make a continuous translation of $\mathbb{T}$ to find a number $\tau_{1}$ such that $\mathbb{T} \cap \mathbb{T}^{\tau_{1}}:=\mathbb{T}_{1}$ is the maximum. Next, consider a translation of $\mathbb{T}_{1}$ again to find a number $\tau_{2}$ such that $\mathbb{T}_{1} \cap \mathbb{T}_{1}^{\tau_{2}}:=\mathbb{T}_{2}$ is the maximum. Continue this process $n$ times to find a number $\tau_{n}$ such that $\mathbb{T}_{n-1} \cap \mathbb{T}_{n-1}^{\tau_{n}}:=\mathbb{T}_{n}$ is the maximum. This process leads to a decreasing sequence of time scale sets:

$$
\mathbb{T} \supset \mathbb{T}_{1} \supset \mathbb{T}_{2} \supset \cdots \supset \mathbb{T}_{n} \supset \cdots
$$

Hence, it follows that $\lim _{n \rightarrow \infty} \mathbb{T}_{n}=\mathbb{T}_{0}^{1}$. This shows that there exists some $\tau_{0}^{1}$ such that $\mathbb{T}_{0}^{1}$ coincides with itself through the translation of this number, obviously, this also implies that $\mathbb{T}_{0}^{1}$ is not a finite union of the closed intervals.

Now we claim that $\mathbb{T}_{0}^{1} \neq \emptyset$. In fact, if $\mathbb{T}_{0}^{1}=\emptyset$, then there exists some sufficiently large $n_{0}$ such that $\mathbb{T}_{n_{0}}=\emptyset$, i.e., there exists some sub-timescale $\mathbb{T}_{n_{0}-1} \subset \mathbb{T}$ such that $\mathbb{T}_{n_{0}-1}$ has no intersection with itself through the translation of the number $\tau_{n_{0}}$ and $\mathbb{T}_{n_{0}-1} \cap \mathbb{T}_{n_{0}-1}^{\tau_{n_{0}}}$ is the maximum element in

$$
\mathbb{I}_{n_{0}}=\left\{\mathbb{T}_{n_{0}-1} \cap \mathbb{T}_{n_{0}-1}^{\tau}: \tau \neq 0\right\}=\emptyset
$$

which means that $\mathbb{T}_{n_{0}-1}$ is a single point set, but this is a contradiction since sup $\mathbb{T}_{n_{0}-1}=$ $+\infty$, inf $\mathbb{T}_{n_{0}-1}=-\infty$. Therefore, $\mathbb{T}_{0}^{1}$ is a $\tau_{0}^{1}$-periodic sub-timescale, that is, $\mathbb{T}_{0}^{1}$ is an invariant under a translation unit in $\mathbb{T}$. This completes the proof.

Example 2.5 Let $a>1$, and consider the following time scale:

$$
\mathbb{P}_{a,|\sin t+\sin \sqrt{5} t|}=\bigcup_{m=1}^{\infty}\left[p_{m}, a+p_{m}\right]
$$

where

$$
\begin{aligned}
p_{m}= & (m-1) a+\sum_{k=1}^{m-1} \mid \sin (k a+|\sin a+\sin \sqrt{5} a| \\
& +\mid \sin (2 a+|\sin a+\sin \sqrt{5} a|) \\
& +\sin \sqrt{5}(2 a+|\sin a+\sin \sqrt{5} a|) \mid \\
& +\cdots+\mid \sin ((k-1) a+|\sin a+\sin \sqrt{5} a|) \\
& +\sin \sqrt{5}((k-1) a+|\sin a+\sin \sqrt{5} a|) \mid) \\
& +\sin \sqrt{5}(k a+|\sin a+\sin \sqrt{5} a| \\
& +\mid \sin (2 a+|\sin a+\sin \sqrt{5} a|) \\
& +\sin \sqrt{5}(2 a+|\sin a+\sin \sqrt{5} a|) \mid
\end{aligned}
$$




$$
\begin{aligned}
& +\cdots+\mid \sin ((k-1) a+|\sin a+\sin \sqrt{5} a|) \\
& \underbrace{+\sin \sqrt{5}((k-1) a+|\sin a+\sin \sqrt{5} a|) \mid) \mid}_{k \text { terms }} .
\end{aligned}
$$

It follows that

$$
\sigma(t)= \begin{cases}t, & \text { if } t \in \bigcup_{m=1}^{\infty}\left[p_{m}, a+p_{m}\right), \\ t+|\sin t+\sin \sqrt{5} t|, & \text { if } t \in \bigcup_{m=1}^{\infty}\left\{a+p_{m}\right\}\end{cases}
$$

and

$$
\mu(t)= \begin{cases}0, & \text { if } t \in \bigcup_{m=1}^{\infty}\left[p_{m}, a+p_{m}\right), \\ |\sin t+\sin \sqrt{5} t|, & \text { if } t \in \bigcup_{m=1}^{\infty}\left\{a+p_{m}\right\} .\end{cases}
$$

For this time scale, since $\mu(t) \leq 2$, it follows from Theorem 2.4 that $\mathbb{T}$ contains at least one invariant under a translation unit.

In view of Theorem 2.1, we can introduce the following concept.

Definition 2.2 Let $\mathbb{T}$ be an arbitrary time scale. If all conditions of Theorem 2.1 are satisfied, then we say the invariant under a translation time scale $\mathbb{T}_{0 *}$ is a sub-invariant under the translation unit in $\mathbb{T}$.

Remark 2.6 It follows from Theorem 2.1 that if we define functions on $\mathbb{T}_{0 *}$, then it will lead to the concept of functions invariant under translation time scales. Since $\mathbb{T}_{0 *}$ is a subset of $\mathbb{T}$ (in other words, $\mathbb{T}_{0 *}$ is a local part of $\mathbb{T}$ ), it opens up a new avenue to investigate the local properties of functions on an arbitrary time scale. Further, we can introduce the concept of local-almost periodic functions on time scales, then local-almost periodic solutions of dynamic equations on time scales can be studied exactly as in [8]. In fact, all the results established in [8] can also be obtained for $\mathbb{T}_{0 *}$ by simply replacing invariant under translation time scales $\mathbb{T}$ in $[8]$ by $\mathbb{T}_{0 *}$ (since $\mathbb{T}_{0 *}$ is a sub-invariant under the translation unit).

Using Remark 2.6, we can give a generalization of Definition 1.8 so called local-uniformly almost periodic functions on time scales.

Definition 2.3 Let $\mathbb{T}$ be an arbitrary time scale. $\mathbb{T}_{0 *}$ is an sub-invariant under the translation unit in $\mathbb{T}$ and $\hat{\Pi}$ the invariant translation set for $\mathbb{T}_{0 *}$. A function $f \in C\left(\mathbb{T} \times D, \mathbb{E}^{n}\right)$ is called a local-uniformly almost periodic function in $t \in \mathbb{T}$ uniformly for $x \in D$ if the $\varepsilon$-translation set of $f$,

$$
E\{\varepsilon, f, S\}=\left\{\tau \in \hat{\Pi} \subset \tilde{\Pi}:|f(t+\tau, x)-f(t, x)|<\varepsilon, \text { for all }(t, x) \in \mathbb{T}_{0 *} \times S\right\}
$$

is a relatively dense set in $\hat{\Pi}$ for all $\varepsilon>0$ and for each compact subset $S$ of $D$, that is, for any given $\varepsilon>0$ and each compact subset $S$ of $D$, there exists a constant $l(\varepsilon, S)>0$ such that each interval of length $l(\varepsilon, S)$ contains a $\tau(\varepsilon, S) \in E\{\varepsilon, f, S\}$ such that

$$
|f(t+\tau, x)-f(t, x)|<\varepsilon, \quad \text { for all }(t, x) \in \mathbb{T}_{0 *} \times S .
$$


Here, $\tau$ is called the $\varepsilon$-local translation number of $f$ and $l(\varepsilon, S)$ is called the local inclusion length of $E\{\varepsilon, f, S\}$.

Remark 2.7 From Definition 2.3, we know that if $f(t)$ is a local-uniformly almost periodic functions on $\mathbb{T}$, then $f(t)$ is uniformly almost periodic on $\mathbb{T}_{0 *}$.

Following [8, 9], we can also give a generalization of Definition 1.1, namely, we can introduce the concept of invariance under translation time scales with respect to a family of sets.

Definition 2.4 Let $\mathfrak{C}$ be a collection of sets which is constructed by subsets of $\mathbb{R}$. A time scale $\mathbb{T}$ is called an invariant under a translation time scale with respect to $\mathfrak{C}$ if

$$
\mathfrak{C}^{*}=\left\{\tau \in \bigcap_{c \in \mathfrak{C}} c: t \pm \tau \in \mathbb{T}, \forall t \in \mathbb{T}\right\} \notin\{\{0\}, \varnothing\}
$$

and $\mathfrak{C}^{*}$ is called the smallest invariant translation set of $\mathbb{T}$ with respect to $\mathfrak{C}$.

Finally, we note that the assumption $\mathfrak{C}^{*}=\{0\}$ makes all results obtained in [9] false. In fact, the following revision is needed.

Definition 2.5 (Correction and generalization of Definition 2.6 in [9]) Let $\mathbb{T}$ be an invariant under a translation time scale with respect to $\mathfrak{C}$. A function $f \in C\left(\mathbb{T} \times D, \mathbb{E}^{n}\right)$ is called an almost periodic function in $t \in \mathbb{T}$ uniformly for $x \in D$ if the $\varepsilon$-translation set of $f$,

$$
E\{\varepsilon, f, S\}=\left\{\tau \in \mathfrak{C}^{*}:|f(t+\tau, x)-f(t, x)|<\varepsilon, \text { for all }(t, x) \in \mathbb{T} \times S\right\}
$$

is a relatively dense set in $\mathfrak{C}^{*}$ for all $\varepsilon>0$ and for each compact subset $S$ of $D$, that is, for any given $\varepsilon>0$ and each compact subset $S$ of $D$, there exists a constant $l(\varepsilon, S)>0$ such that each interval of length $l(\varepsilon, S)$ contains a $\tau(\varepsilon, S) \in E\{\varepsilon, f, S\}$ such that

$$
|f(t+\tau, x)-f(t, x)|<\varepsilon, \quad \text { for all }(t, x) \in \mathbb{T} \times S .
$$

Here, $\tau$ is called the $\varepsilon$-translation number of $f$ and $l(\varepsilon, S)$ is called the inclusion length of $E\{\varepsilon, f, S\}$.

To conclude we emphasize that the precise definition of almost (or local-almost) periodic functions on time scales occupies a fundamental position in establishing some key results for dynamical systems on time scales. Thus, the above corrections not only fill the gaps in the existing literature, but they will also help to pursue further research in the right direction. 


\section{Author details}

${ }^{1}$ Department of Mathematics, Yunnan University, Kunming, Yunnan 650091, People's Republic of China. ${ }^{2}$ Department of Mathematics, Texas A\&M University-Kingsville, 700 University Blvd., Kingsville, TX 78363-8202, USA. ${ }^{3}$ Department of Mathematics, Faculty of Science, King Abdulaziz University, P.O. Box 80203, Jeddah, 21589, Saudi Arabia.

\section{Acknowledgements}

The authors would like to express their sincere thanks to the referees for suggesting some corrections that helped making the content of the paper more accurate. This work is supported by Yunnan University Scientific Research Fund Project of China (No. 2013CG020), Yunnan Province Education Department Scientific Research Fund Project of China (No. 2014Y008), and Yunnan Province Science and Technology Department Applied Basic Research Project of China (No. 2014FB102).

Received: 9 June 2015 Accepted: 28 September 2015 Published online: 09 October 2015

\section{References}

1. Dogan, A, Graef, JR, Kong, L: Higher order singular multipoint boundary value problems on time scales. Proc. Edinb. Math. Soc. 54, 345-361 (2011)

2. Dogan, A: Existence of multiple positive solutions for $p$-Laplacian multipoint boundary value problems on time scales. Adv. Differ. Equ. 2013, 238 (2013)

3. Wang, C, Agarwal, RP: Changing-periodic time scales and decomposition theorems of time scales with applications to functions with local almost periodicity and automorphy. Adv. Differ. Equ. 2015, 296 (2015)

4. Dogan, A: Triple positive solutions for $m$-point boundary-value problems of dynamic equations on time scales with p-Laplacian. Electron. J. Differ. Equ. 2015, 131 (2015)

5. Wang, C: Almost periodic solutions of impulsive BAM neural networks with variable delays on time scales. Commun. Nonlinear Sci. Numer. Simul. 19, 2828-2842 (2014)

6. Wang, C, Agarwal, RP: A classification of time scales and analysis of the general delays on time scales with applications. Math. Methods Appl. Sci. (2015). doi:10.1002/mma.3590

7. Wang, C, Agarwal, RP: Uniformly rd-piecewise almost periodic functions with applications to the analysis of impulsive $\Delta$-dynamic system on time scales. Appl. Math. Comput. 259, 271-292 (2015)

8. Li, Y, Wang, C: Uniformly almost periodic functions and almost periodic solutions to dynamic equations on time scales. Abstr. Appl. Anal. 2011, Article ID 341520 (2011)

9. Li, Y, Wang, C: Almost periodic functions on time scales and applications. Discrete Dyn. Nat. Soc. 2011, Article ID 727068 (2011)

10. Liang, T, Yang, Y, Liu, Y, Li, L: Existence and global exponential stability of almost periodic solutions to Cohen-Grossberg neural networks with distributed delays on time scales. Neurocomputing 123, 207-215 (2014)

11. Li, Y, Wang, C: Almost periodic solutions of shunting inhibitory cellular neural networks on time scales. Commun. Nonlinear Sci. Numer. Simul. 17, 3258-3266 (2012)

12. Zhi, Y, Ding, Z, Li, Y: Permanence and almost periodic solution for an enterprise cluster model based on ecology theory with feedback controls on time scales. Discrete Dyn. Nat. Soc. 2013, Article ID 639138 (2013)

13. $\mathrm{Hu}, \mathrm{M}, \mathrm{Xie}, \mathrm{P}$ : Almost periodic solutions of neutral delay functional differential equations on time scales. Bull. Malays Math. Soc. 38, 317-331 (2015)

14. Gao, J, Wang, Q, Zhang, L: Existence and stability of almost-periodic solutions for cellular neural networks with time-varying delays in leakage terms on time scales. Appl. Math. Comput. 237, 639-649 (2014)

15. Zhou, H, Zhou, Z, Jiang, W: Almost periodic solutions for neutral type BAM neural networks with distributed leakage delays on time scales. Neurocomputing 157, 223-230 (2015)

16. Liao, Y, Xu, L: Almost periodic solution for a delayed Lotka-Volterra system on time scales. Adv. Differ. Equ. 2014,96 (2014)

17. Li, L, Li, Y, Yang, L: Almost periodic solutions for neutral delay Hopfield neural networks with time-varying delays in the leakage term on time scales. Adv. Differ. Equ. 2014, 178 (2014)

18. Li, Y, Wang, P: Permanence and almost periodic solution of a multispecies Lotka-Volterra mutualism system with time varying delays on time scales. Adv. Differ. Equ. 2015, 230 (2015)

19. Lizama, C, Mesquita, JG: Almost automorphic solutions of dynamic equations on time scales. J. Funct. Anal. 265, 2267-2311 (2013)

20. Fink, AM: Almost Periodic Differential Equations. Lecture Notes in Mathematics, vol. 377. Springer, Berlin (1974)

21. Bohner, M, Peterson, A: Dynamic Equations on Time Scales: An Introduction with Applications. Birkhäuser, Boston (2001)

22. Wilansky, A: Topics in Functional Analysis. Lecture Notes in Mathematics, vol. 45. Springer, Berlin (1967) 